\title{
Jet quenching as a probe of gluon plasma formation
}

\author{
G. Fai' ${ }^{* 1}$, G.G. Barnaföldi ${ }^{2}$, M. Gyulassy ${ }^{3}$, P. Lévai ${ }^{2}$, G. Papp ${ }^{4}$, I. Vitev ${ }^{3}$ and Y. Zhang ${ }^{1}$ \\ ${ }^{1}$ CNR, Dept. of Physics, Kent State Univ., Kent, OH 44242, USA \\ ${ }^{2}$ KFKI RMKI, P.O. Box 49, Budapest 1525, Hungary \\ ${ }^{3}$ Dept. of Physics, Columbia Univ., 538 W. 120-th Street, New York, NY 10027, USA \\ ${ }^{4}$ HAS Research Group for Theoretical Physics, P.O. Box 32, Budapest 1518, Hungary \\ E-mail: ifai@ecnrred.kent.edù
}

ABSTRACT: We study, in a pQCD calculation augmented by nuclear effects, the jet energy loss needed to reproduce the $\pi^{0}$ spectra in $\mathrm{Au}+\mathrm{Au}$ collisions at large $p_{T}$, measured by PHENIX at RHIC. The transverse width of the parton momentum distributions (intrinsic $k_{T}$ ) is used phenomenologically to obtain a reliable baseline $p p$ result. Jet quenching is applied to the nuclear spectra (including shadowing and multiscattering) to fit the data.

KEYwords: $\mathrm{pQQ} \bar{C} \overline{\mathrm{D}}$, high $p_{T}$, intrinsic transverse momentum, jet quenching.

\section{Introduction}

The average energy loss of jets in central $A u+A u$ collisions at RHIC can be used to estimate the density of gluons in the medium. To determine the effect of jet energy loss from the data, one needs a reliable background calculation of pion and kaon spectra subject to jet quenching. Here we provide the required baseline pQCD calculation augmented by the nuclear effects of multiscattering and shadowing.

\section{Parton model and $p Q C D$ with intrinsic transverse momentum}

The invariant cross section for the production of hadron $h$ in a $p p$ collision is described in the pQCD-improved parton model on the basis of the factorization theorem as a convolution [i]1]:

$E_{h} \frac{\mathrm{d} \sigma_{h}^{p p}}{\mathrm{~d}^{3} p}=\sum_{a b c d} \int \mathrm{d} x_{a, b} \mathrm{~d} z_{c} f_{a / p}\left(x_{a}, k_{T a}, Q^{2}\right) f_{b / p}\left(x_{b}, k_{T b}, Q^{2}\right) \frac{\mathrm{d} \sigma}{\mathrm{d} \hat{t}} \frac{D_{h / c}\left(z_{c}, Q^{\prime 2}\right)}{\pi z_{c}^{2}} \hat{s} \delta(\hat{s}+\hat{t}+\hat{u})$,

where $f_{a, b / p}\left(x, k_{T}, Q^{2}\right)$ are the parton distribution functions (PDFs) for the colliding partons $a$ and $b$ in the interacting protons as functions of momentum fraction $x$, at scale $Q$,

${ }^{*}$ Speaker. 
incorporating a Gaussian transverse momentum distribution. Having convinced ourselves that it makes sense to use a $p_{T}$-independent width at a given energy [2i], we treat the width of the transverse momentum distribution, $\left\langle k_{T}^{2}\right\rangle_{p p}$, as an energy-dependent parameter (also see e.g. [isi]).

In eq. $(\overline{2} \cdot \overline{1}), \mathrm{d} \sigma / \mathrm{d} \hat{t}$ is the hard scattering cross section of the partonic subprocess $a b \rightarrow c d$, and $D_{h / c}\left(z_{c}, Q^{\prime 2}\right)$, the fragmentation function $(\mathrm{FF})$, gives the probability for parton $c$ to fragment into hadron $h$ with momentum fraction $z_{c}$ at scale $Q^{\prime}$. The scales are fixed in the present work as $Q=p_{T} / 2$ and $Q^{\prime}=p_{T} /\left(2 z_{c}\right)$. We use leading order (LO)

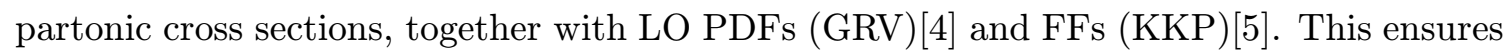
the consistency of the calculation. Approximate NLO treatments via a $p_{T}$ and energy independent "K factor" are also possible $\left[\overline{6}_{1}^{1}, \overline{i_{1}}\right]$. . However, the dependence of the $\mathrm{K}$ factor on $p_{T}$ and $\sqrt{s}$ can not be neglected if a wide range of these variables is to be addressed[s]. In addition, we prefer to keep the effects of the $\mathrm{K}$ factor separate from those of the intrinsic transverse momentum.

Fig. 1 displays the extracted values of the width $\left\langle k_{T}^{2}\right\rangle_{p p}$ from hard pion production data at energies below $\sqrt{s} \lesssim 60 \mathrm{GeV}$ and from $h^{ \pm}=\left(h^{+}+h^{-}\right) / 2$ data at higher energies. The solid curve is drawn to guide the eye, while the dashed lines delineate an estimated band of increasing uncertainty with increasing $\sqrt{s}$. We use this band to read off the $\left\langle k_{T}^{2}\right\rangle_{p p}$ values to be applied at RHIC energies. The solid bars represent estimates at $\sqrt{s}=130$ and $200 \mathrm{GeV}$, respectively. We use $\left\langle k_{T}^{2}\right\rangle_{p p}=2$ $\mathrm{GeV}^{2}$ in our pQCD calculations analyzing recent RHIC results at $\sqrt{s}=130 \mathrm{AGeV}$.

\section{Nuclear shadowing and multiscattering}

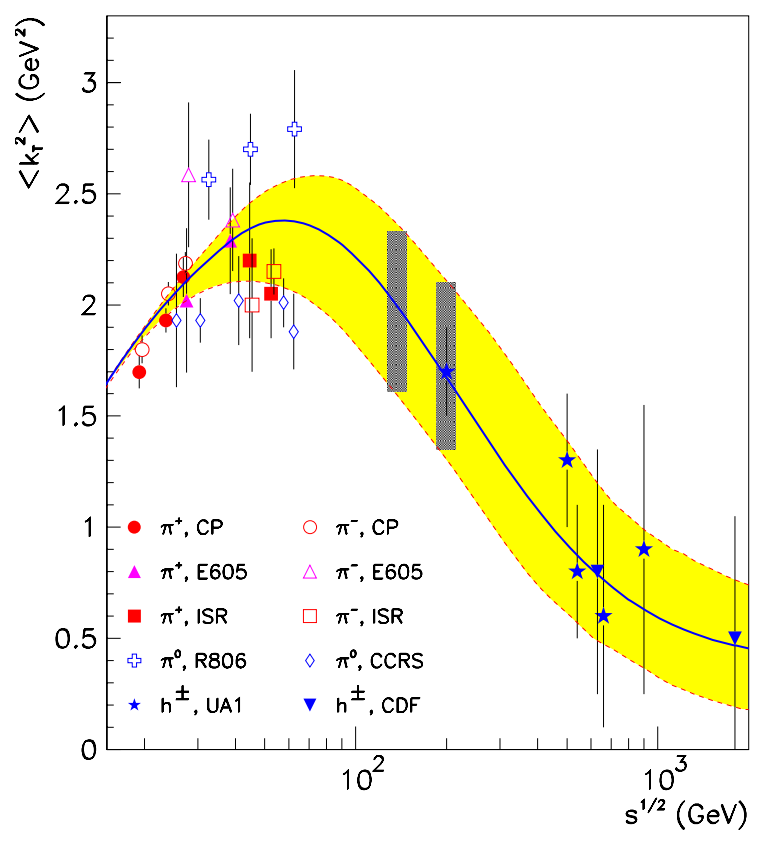

We approximately include the modification of the PDFs inside nuclei

Figure 1: The best fit values of $\left\langle k_{T}^{2}\right\rangle_{p p}$ in $p p \rightarrow \pi X$ and $p \bar{p} \rightarrow h^{ \pm} X$ reactions. Where large error bars would overlap at the same energy, one of the points has been shifted slightly for better visibility. The band is drawn to guide the eye.

("shadowing") and the isospin asymmetry of heavy nuclei into the nuclear PDFs via the average nuclear dependence and a scale independent shadowing function $S_{a / A}(x)$ adopted from $\left[\begin{array}{l}\overline{9} \\ \underline{9}-1\end{array}\right]$ :

$$
f_{a / A}\left(x, Q^{2}\right)=S_{a / A}(x)\left[\frac{Z}{A} f_{a / p}\left(x, Q^{2}\right)+\left(1-\frac{Z}{A}\right) f_{a / n}\left(x, Q^{2}\right)\right]
$$


where $f_{a / n}\left(x, Q^{2}\right)$ is the PDF for the neutron.

The enhancement of the width of the transverse momentum distribution of partons due to interactions in the medium ("multiscattering") is treated in the Glauber framework [i] We hold multiscattering accountable for the Cronin effect in $p A$ collisions, and use $p A$ data to parameterize the width enhancement. The enhanced width is written as

$$
\left\langle k_{T}^{2}\right\rangle_{p A}=\left\langle k_{T}^{2}\right\rangle_{p p}+C \cdot\left(\nu_{A}(b)-1\right),
$$

where $\left\langle k_{T}^{2}\right\rangle_{p p}$ is the width of the parton transverse momentum distribution in $p p$ collisions, $\nu_{A}(b)-1$ is the number of effective nucleon-nucleon $(\mathrm{NN})$ collisions at impact parameter $b$, which impart an average transverse momentum squared $C$. In $p A$ reactions, where one of the hard-colliding partons originates in a nucleon with additional NN collisions, we use the $p p$ width from Fig. 1 for one of the colliding partons and the width (i3.2.

$$
E_{h} \frac{\mathrm{d} \sigma_{h}^{p A}}{\mathrm{~d}^{3} p}=\int \mathrm{d}^{2} b t_{A}(b) E_{h} \frac{\mathrm{d} \sigma_{h}^{p p}\left(\left\langle k_{T}^{2}\right\rangle_{p A},\left\langle k_{T}^{2}\right\rangle_{p p}\right)}{\mathrm{d}^{3} p},
$$

where $t_{A}(b)=\int \mathrm{d} z \rho(b, z)$ is the nuclear thickness function normalized as $\int \mathrm{d}^{2} b t_{A}(b)=A$. We obtain the best fit to the Cronin data if we maximize the number of effective NN collisions at a given impact parameter at $\left(\nu_{A}(b)-1\right)_{\max }=3$, and take the value of the parameter $C=0.4 \mathrm{GeV}^{2}$.

\section{Jet quenching}

In $A A$ reactions, where both hard-colliding partons originate in nucleons with additional semi-hard collisions, we use the width $\left(\overline{3}, \overline{2}_{2}\right)$ for both initial partons. Thus,

$$
E_{h} \frac{\mathrm{d} \sigma_{h}^{A B}}{\mathrm{~d}^{3} p}=\int \mathrm{d}^{2} b \mathrm{~d}^{2} r t_{A}(r) t_{B}(|\vec{b}-\vec{r}|) E_{h} \frac{\mathrm{d} \sigma_{h}^{p p}\left(\left\langle k_{T}^{2}\right\rangle_{p A},\left\langle k_{T}^{2}\right\rangle_{p B}\right)}{\mathrm{d}^{3} p},
$$

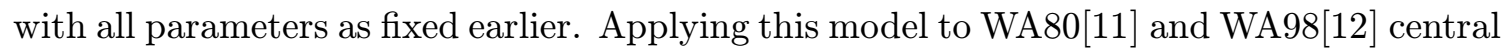
$\pi^{0}$ production data at SPS energies, we find a reasonable agreement beyond a transverse momentum of $p_{T} \gtrsim 2-2.5 \mathrm{GeV}$, with possibly an overestimation of the data by the model in the heaviest system $(P b+P b)$ by upto $40 \%[2]$. This may be taken as a hint that an additional mechanism is at work in the nuclear medium, which acts to reduce the calculated cross sections. As we will see next, the discrepancy becomes more serious at RHIC energies. Since this effect can also be looked upon as a shift of the spectra to lower $p_{T}$, i.e. jet energy

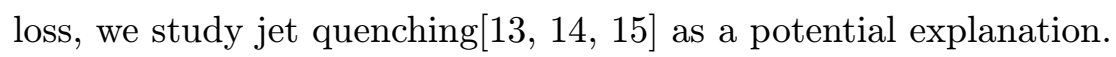

Assuming a static gluon plasma, we use here the average energy loss of gluon jets to

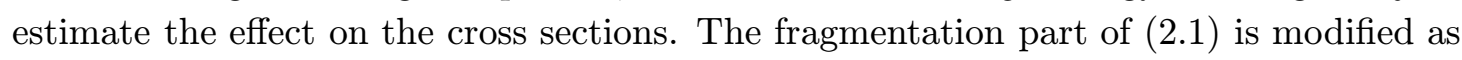

$$
\frac{D_{h / c}\left(z_{c}, Q^{\prime 2}\right)}{\pi z_{c}^{2}} \longrightarrow \frac{z_{c}^{*}}{z_{c}} \frac{D_{h / c}\left(z_{c}^{*}, Q^{\prime 2}\right)}{\pi z_{c}^{2}},
$$

where the down-shifted (quenched) momentum $p_{c}^{*}=p_{c}-\Delta E$ results in a momentum fraction $z_{c}^{*}=z_{c} /\left(1-\Delta E / p_{c}\right)$. For the energy shift $\Delta E$ we use the "thin plasma" (GLV) 
approximation[1] [i] $\left.\overline{5}_{1}\right]$, appropriate for small values of the opacity $\bar{n}=L / \lambda$, where $L$ is the average thickness of the static medium and $\lambda$ denotes the gluon mean free path. One important feature distinguishing the GLV energy loss from the "thick plasma" (BDMS) limit [i] [i], is the approximately constant fractional energy loss $(\Delta E / E)$ of gluons as a function of jet energy $E$ in the range $3 \lesssim E \lesssim 10 \mathrm{GeV}\left[\overline{1} \overline{1} \bar{j}_{1}\right.$.

In Fig. ${ }_{2}^{2}$ the results of this calculation are compared to recent $\pi^{0}$ spec-

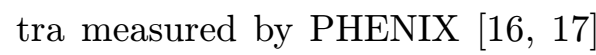
for values of the opacity $0 \leq \bar{n} \leq 4$. It can be seen that, while the semiperipheral spectrum displays only a weak (but non-vanishing) influence of jet-quenching characterized by $\bar{n} \approx 1-1.5$, in the central case a much larger effective opacity of $\bar{n} \approx$ $3-4$ is required for agreement between the data and pQCD calculations [1 $1 \overline{2} \overline{1}$ ]. It should be noted that fluctuations in jet energy loss reduce the suppression, but their net effect can be absorbed in a rescaling of the opacity $\bar{n}$. First results on an approximate treatment of fluctuations and on a dynamical calculation are reported in the next talk [i] $\underline{1} \overline{9}]$.

The average opacity $\bar{n}$ can be related to the density of the plasma using the expression of the transport coefficient in terms of $\mathrm{d} N_{g} / \mathrm{d} y$ [1] plies a very high initial gluon density for $\bar{n} \approx 3$ in a static plasma. Taking into account the rapid trans-

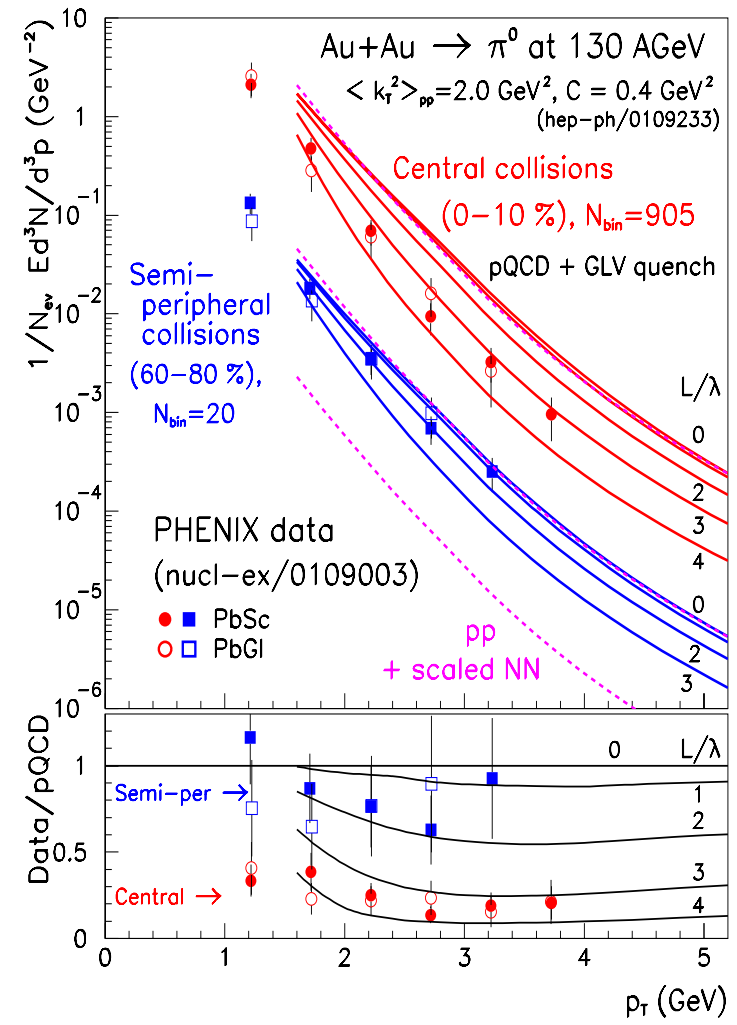

Figure 2: Semi-peripheral and central $\pi^{0}$ production in $A u+A u$ collisions at $\sqrt{s}=130 \mathrm{GeV}$. Data are from Ref. [1 $\overline{7}_{1}$. Calculation with jet quenching at opacities $L / \lambda=0,1,2,3,4$. Dashed lines are $p p \rightarrow \pi^{0}$ and Glauberscaled estimates for $\mathrm{Au}+\mathrm{Au}$. verse collective expansion of the plasma leads to somewhat reduced gluon densities, $\mathrm{d} N_{g} / \mathrm{d} y \approx$ 500 , and to results on the collective flow consistent with the preliminary data[20 [20 - By any reasonable estimate these gluon densities indicate that strongly-interacting matter has reached the deconfined region in the analyzed nuclear collisions.

\section{Summary and conclusions}

Jet quenching is needed on the background of a pQCD calculation augmented by the nuclear effects of shadowing and multiscattering to bring the calculated results in agreement with the PHENIX data on $\pi^{0}$ spectra from $A u+A u$ at $\sqrt{s}=130 \mathrm{GeV}$. The amount of required 
jet energy loss indicates a dense gluon plasma, well in the deconfined region. Jet quenching is a powerful diagnostic tool to study strongly-interacting matter in nuclear collisions, which will play an important role in analyzing the $2001 \mathrm{RHIC}$ data at $\sqrt{s}=200 \mathrm{GeV}$, and beyond.

\section{Acknowledgments}

We thank G. David for discussions. This work was supported in part by U.S. NSF grant INT-0000211 (MTA-OTKA-NSF), DOE grant DE-FG02-86ER40251, and Hungarian grants FKFP220/2000, OTKA-T032796, OTKA-T034269 and OTKA-T034842.

\section{References}

[1] R.D. Field, Applications of Perturbative QCD, Addison-Wesley, 1995.

[2] Y. Zhang, G. Fai, G. Papp, G.G. Barnaföldi and P. Lévai, hep-ph/0109233 (2001).

[3] M.D. Corcoran et al. (E609), Phys. Lett. B 259, 209 (1991).

[4] M. Glück, E. Reya, and A. Vogt, Z. Phys. C 53, 127 (1992).

[5] B.A. Kniehl, G. Kramer, and B. Pötter, Nucl. Phys. B 597, 337 (2001).

[6] X.N. Wang, Phys. Rev. C 61, 064910 (2001); C.Y. Wong and H. Wang, Phys. Rev. C 58, 376 (1998).

[7] G. Papp, P. Lévai, and G. Fai, Phys. Rev. C 61, 021902(R) (2000).

[8] G.G. Barnaföldi, G. Fai, P. Lévai, G. Papp, and Y. Zhang, J. Phys. G 27, 1767 (2001).

[9] X.N. Wang and M. Gyulassy, Phys. Rev. D 44, 3501 (1991).

[10] R.J. Glauber, in Lectures in Theoretical Physics, ed. W.E Brittin and L.G. Dunham, Interscience, N.Y., Vol. 1, p315 (1959); R.J. Glauber and G. Matthiae, Nucl. Phys. B 21, 135 (1970); V.N. Gribov, JETP 30, 709 (1970).

[11] R. Albrecht et al. (WA80), Phys. Lett. B 361, 14 (1995).

[12] M.M. Aggarwal et al. (WA98), Phys. Rev. Lett. 85, 3595 (2000); nucl-ex/0006007; nucl-ex/0108006 (2001).

[13] M. Gyulassy and M. Plümer, Phys. Lett. B 243, 432 (1990); X.-N. Wang and M. Gyulassy, Phys. Rev. Lett. 68, 1480 (1992).

[14] R. Baier, Yu.L. Dokshitzer, A.H. Mueller, and D. Schiff, Nucl. Phys. B 531, 403 (1998); R. Baier, D. Schiff, B.G. Zakharov, Ann. Rev. Nucl. Part. Sci. 50, 37 (2000).

[15] M. Gyulassy, P. Lévai, and I. Vitev, Phys. Rev. Lett. 85, 5535 (2000); Nucl. Phys. B 594, 371 (2001).

[16] G. David et al. (PHENIX), Nucl. Phys. A698, 227 (2002), nucl-ex/0105014.

[17] K. Adcox et al. (PHENIX), nucl-ex/0109003 (2001).

[18] P. Lévai, G. Papp, G. Fai, M. Gyulassy, G.G. Barnaföldi, I. Vitev, Y. Zhang, Nucl. Phys. A698, 631 (2002), nucl-th/0104035.

[19] I. Vitev, M. Gyulassy, and P. Lévai, JHEP PRHEP-hep2001/243, hep-ph/0109198.

[20] M. Gyulassy, I. Vitev, X-N. Wang, and P. Huovinen, nucl-th/0109063 (2001). 